\author{
В. Л. ГРЕШТА ${ }^{1}$, Д. В. ПАВЛЕНКО ${ }^{1,2}$, Я. В. ДВИРНЫК ${ }^{3}$, Д. В. ТКАЧ 1 \\ 1 Запорожский национальный технический университет, Запорожье, Украина \\ 2 ГП «Ивченко-Прогресс», Запорожье, Украина \\ ${ }^{3}$ АО «МОТОР СИЧ», Запорожье, Украина
}

\title{
РАСЧЕТНО-ЭКСПЕРИМЕНТАЛЬНАЯ МЕТОДИКА ОПРЕДЕЛЕНИЯ ДИНАМИЧЕСКОГО МОДУЛЯ УПРУГОСТИ ПРИРАБАТЫВАЕМЫХ УПЛОТНИТЕЛЬНЫХ ПОКРЫТИЙ ТУРБИН ГТД
}

\begin{abstract}
Целью работы была разработка и апробаџия методики определения динамического модуля упругости прирабатываемых уплотнительных покрытий турбин газотурбинных двигателей (ГТД). К данньлм покрытиям выдвигается ряд противоречивых требований, поэтому для их удовлетворения предложено применять покрытия с переменными свойствами на различных этапах жизненного цикла газотурбинных двигателей. Однако разработка новых покрытий требует проведения различного рода механических испьтаний, в том числе и для оценки динамического модуля упругости. Пористая структура u, соответственно, низкая прочность разрабатываемых покрытий не позволяет применять стандартные методы оценки механических свойств, поэтому возникла необходимость разработки специальной методики определения модуля упругости. В процессе исследования были применены метод конечных элементов, статистические методы, экспериментальные методы определения собственной частоты колебаний. Исследования выполняли для прирабатываемого уплотнительного покрытия статора турбин газотурбинных двигателей КНA-82+CoNiCrAlY. Численный эксперимент был выполнен в программном комплексе Ansys Workbench 2019 R2. Поскольку покрытия применяются в условиях повышенных температур необходимо было оченивать модуль упругости при различных температурах, что требовало проведения дополнительных исследований температурозависимых свойств, влияющих на искомую величину. В результате реализаџии плана численного эксперимента по определению частоты собственных колебаний образцов, с нанесенным покрытием при варьировании его модуля упругости и температуры, а также решения обратной задачи установления зависимости модуля динамической упругости от частоты собственных колебаний образиа с покрытием, разработали расчетно-экспериментальную методику определения динамического модуля упругости прирабатываемых уплотнительных покрытий турбин ГТД. Разработанная методика применяется для определения динамического модуля упругости прирабатываемых покрытий различного химического состава $и$ структуры в области рабочих температур, что может быть использовано для оптимизации их состава, структуры и свойств.
\end{abstract}

Ключевые слова: план эксперимента; модуль упругости; прирабатываемое покрытие; газотурбинный двигатель; методика; собственная частота; метод конечных элементов; модуль упругости.

\section{Введение}

Прирабатываемые уплотнительные покрытия играют важную роль в конструкции газотурбинных двигателей (ГТД) с точки зрения обеспечения его высокого коэффициента полезного действия. В связи с этим, к ним предъявляется ряд требований, которые в некоторых случаях могут носить взаимно противоречивый характер. Так, например, такие покрытия должны обладать минимальной твердостью, что обеспечит минимальный износ контактирующих с ним гребешков ротора ГТД. Однако, с другой стороны, они должны обладать высокой твердостью, что связано с необходимостью обеспечения их сопротивления эрозионному изнашиванию горячим потоком газов. Компромисс в данном случае может быть найден путем поиска оптимального химического состава покрытия, приводящего к изменению его свойств на различных этапах жизненного цикла ГТД. Во всех случаях одним из важных критериев оптимизации покрытия является его адгезионная прочность с материалом основы. Одним из факторов, ее определяющих является различия в модуле упругости и коэффициентах термического расширения покрытия и основы. Таким образом, поскольку модуль упругости покрытия зависит от 
его состава и структуры то в процессе их оптимизации необходимо иметь надежную методику его определения.

\section{Постановка целей и задач исследования}

Целью исследования являлась разработка и апробация расчетно-экспериментальной методики определения динамического модуля упругости прирабатываемых уплотнительных покрытий турбин ГТД. Для ее достижения были решены задачи, связанные с реализацией проекта численного определения частоты собственных колебаний образца с покрытием при различных температурах в системе ANSYS; разработкой плана численного эксперимента и его проведением; установлением зависимости модуля упругости от частоты собственных колебаний и температуры, а также экспериментальным определением частоты собственных колебаний образцов с покрытием.

\section{Обзор литературы в области методов оценки модуля упругости покрытий}

В процессе разработки новых газотермических уплотнительных покрытий возникает необходимость определять их механические характеристики, что в случае пористых покрытий требует использования методик, отличающихся от применяемых при исследовании стандартных металлов и сплавов. Учитывая, что уплотнительные покрытия работают в условиях динамического нагружения и модуль Юнга является важной характеристикой при прогнозировании поведения деталей в процессе эксплуатации, актуальной задачей является определение данного модуля у разрабатываемых покрытий. Применение стандартной методики измерения модуля упругости на основании получения кривых растяжения ограничено невозможностью получить стандартный образец для исследования. Поэтому ученые предлагают различные альтернативные методы оценки данного модуля: как экспериментальные, так и расчетные. Расчетные методы основаны на построении сложных зависимостей и требуют экспериментального подтверждения [1-3]. Поскольку на свойства покрытий такого типа значительно влияют особенности технологии их получения, то наиболее рационально применять экспериментальные методы оценки модуля упругости. В частности, в работе [4] предложено оценивать такого рода материалы динамическим резонансным методом. Данный метод позволяет оценить величину динамического модуля упругости слоистых покрытий, однако, как сам экс- перимент, так и дальнейший его расчет являются достаточно громоздкими. Методы расчета физикомеханических характеристик покрытия при испытаниях на изгиб предложенные в работах $[5,6]$ менее трудоемки, однако погрешность вносимая часовым индикатором может несколько искажать результаты измерений. Подобный метод оценки модуля Юнга применяли в работе [7], однако он применим лишь в случае объемных пористых материалов, т.к. последующий расчет не учитывает наличия матричного материала. Микро- и наноиндентирование позволяет определять механические характеристики покрытий различной толщины, при этом данный метод применим как на плотных, так и пористых покрытиях [8,9]. В работе [10] указано, что нанесение покрытий на стандартный образец меняет его физикомеханические характеристики, было предложено оценивать модуль упругости покрытия сравнением образцов. Для этого проводились испытания на растяжение образцов с нанесенным покрытием и без него. После чего, путем нетрудоемкого расчета, оценивалась величина модуля Юнга покрытий. В данном случае было необходимо использовать специальное приспособление, которое позволяло высокоточно определять удлинение образцов. Испытания на растяжение для оценки модуля упругости также были применены в работе [11], что позволило оценить также другие характеристики прочности (предел прочности, относительное удлинение). Однако в данном случае также возникла необходимость дополнительной механической обработки образцов, поскольку необходимо было отделять покрытия от подложки. И, следовательно, данный метод позволяет оценивать только достаточно объемные покрытия, либо пористые материалы. В работе [12] оценивался динамический модуль упругости с использованием метода динамической резонансной частоты в режиме изгибных колебаний при различных температурах в диапазоне от 20 до $1000^{\circ} \mathrm{C}$.

Таким образом, большинство предложенных выше методов применимы только для определения статистического модуля упругости. Исходя из анализа существующих методов оценки модуля упругости пористых материалов и различного рода покрытий, для разрабатываемого уплотнительного покрытия целесообразно применять метод динамической резонансной частоты. Однако, разработанное покрытие невозможно испытывать без подложки, поэтому возникает необходимость доработки данной методики.

Целью настоящей работы являлось разработка и апробация расчетно-экспериментальной методики определения динамического модуля упругости прирабатываемых уплотнительных покрытий турбин ГТД. Для ее достижения были решены задачи, свя- 
занные с решением прямой и обратной задачи оценки влияния динамического модуля упругости покрытий при различных температурах на частоту собственных колебаний образца.

\section{Материалы и методы исследования}

Исследования выполняли для прирабатываемого уплотнительного покрытия статора турбин газотурбинных двигателей КНА-82+CoNiCrAlY [13] после термической обработки (рис. 1). Плотность исследуемого прирабатываемого покрытия составляла $3,4 \kappa г / \mathrm{M}^{3}$.

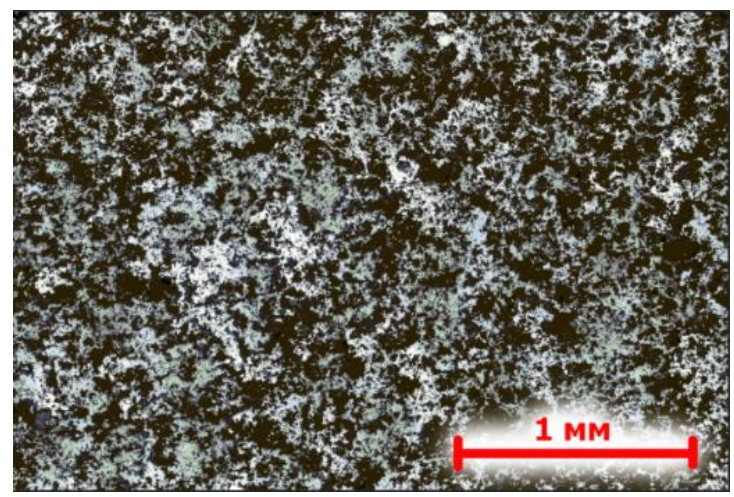

a

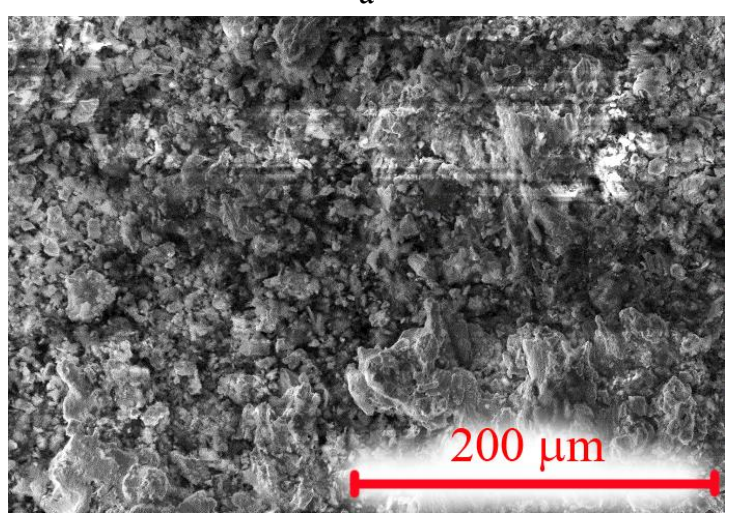

6

Рис. 1. Микроструктуры уплотнительного покрытия KHA-82+CoNiCrAlY: a - оптический микроскоп; б - растровый электронной микроскоп

Для реализации проекта численного эксперимента в программном комплексе Ansys Workbench 2019 R2 использовали модули Geometry и Modal. Учитывая необходимость проведения многократного расчёта частот собственных колебаний образцов с различными значениями модуля упругости уплотнительного покрытия и температуры, использовали модуль планирования эксперимента Design of Experiments (рис. 2). Высокий уровень автоматизации вычислений позволил получить возможность реализации полнофакторного эксперимента при большом числе независимых переменных.
Для проведения численного эксперимента методом конечных элементов (МКЭ) на первом этапе, с помощью CAD системы SpaceClame были построены твердотельные модели исследуемого образца в исходном состоянии (рис. 3 , а) и образца с нанесенным уплотнительным покрытием (рис. 3, б). Определение собственных частот колебаний образца проводили в подпрограмме ANSYS Modal с использованием решателя Mechanical APDL. Покрытие моделировали путем симметричного добавления материала на рабочую часть образца (рис. 4).

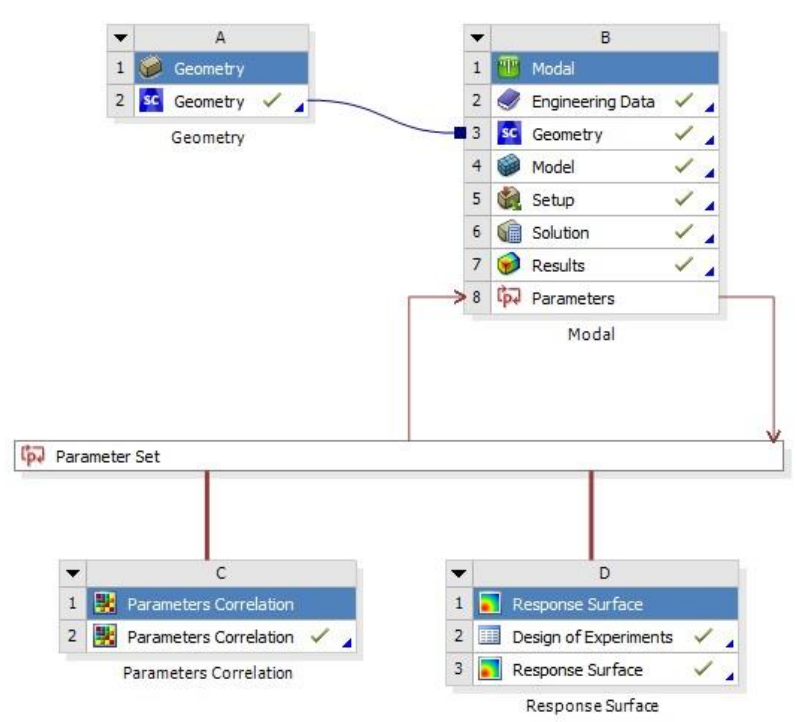

Рис. 2. Схема проекта расчета собственных частот образцов в системе Ansys Workbench 2019 R2

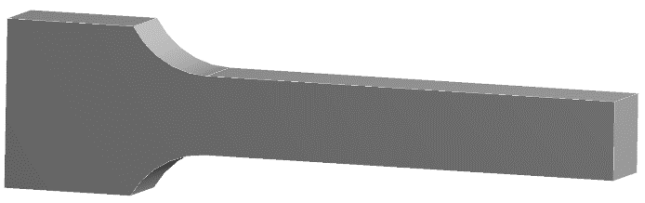

a

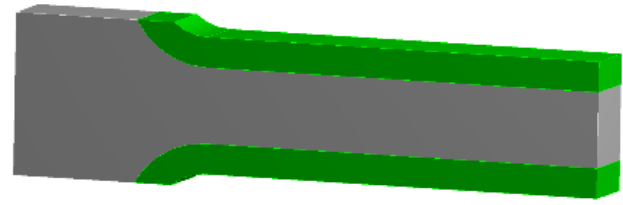

б

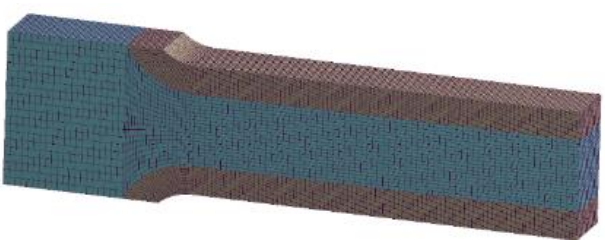

B

Рис. 3. Твердотельная модель образца без покрытия (a), с нанесенным уплотнительным покрытием (б) и конечно-элементная модель образца с нанесенным уплотнительным покрытием (в) 
На основе полученных геометрических моделей строили структурированную расчетную сетку с использованием сеточного генератора ICEM CFD (рис. 3, в). Ячейки полученной стеки имели гексаэдрическую форму. Сеточная модель образца состояла из $120 \ldots 130$ тысяч 20-ти узловых элементов solid186. Применение структурированной гексаэдрической стеки высокого качества имеет ряд преимуществ над другими типами сеток конечных элементов, основным из которых является высокая точность при оптимальном времени счета.

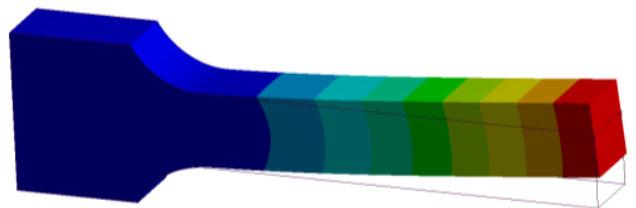

$\mathrm{a}$

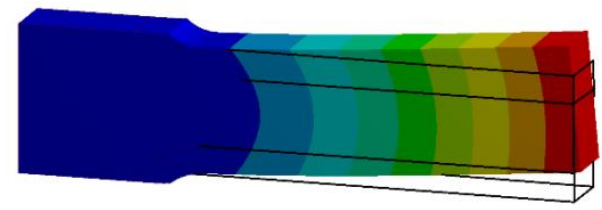

б

Рис. 4. Поля перемещения образца при колебании по второй изгибной форме без покрытия (a) и с покрытием (б)

Температуру образца задавали в виде граничных условий первого рода (значение температуры в узлах конечно-элементной модели). Также ограничивали перемещения во всех направлениях верхней и нижней торцевой поверхности образца, что адекватно моделировало его закрепление на испытательной установке.

Результатом модального анализа являлись значения 3-х первых собственных частот образца.

Исследования выполняли на образцах, изготовленных из жаропрочного сплава ЭП367. Учитывая, что экспериментально собственная частота определяется для системы образец-покрытие, для получения достоверных результатов значений динамического модуля упругости покрытий свойства материала основы задавали зависимыми от температуры. Для установления температурозависимых свойств сплава ЭП367 использовали программный пакет для моделирования свойств сталей и сплавов JMatPro на основе известных его химического состава и термодинамических процессов, протекающих в сплаве. Значения температурного коэффициента линейного расширения покрытия (ТКЛР) определяли на стандартных образцах на дилатометре Шевенара [13].

Учитывая, что установление зависимости изменения плотности и коэффициента Пуассона для материалов уплотнительных покрытий является ме- тодологически сложной задачей на данном этапе моделирования значения указанных величин принимали постоянными, не зависящими от температуры.

Экспериментальное определение собственной частоты изгибных колебаний образцов без покрытий (рис. 5, а) и образцов с нанесенным покрытием KНA-82+CoNiCrAlY (рис. 5, б) выполняли на установке для усталостных испытаний «Микат-У» (рис. 6).

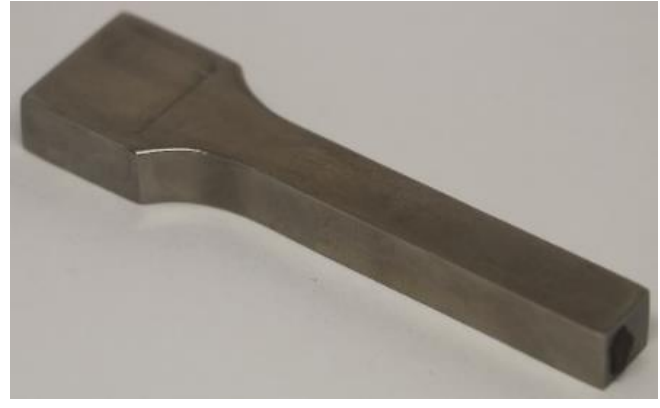

a

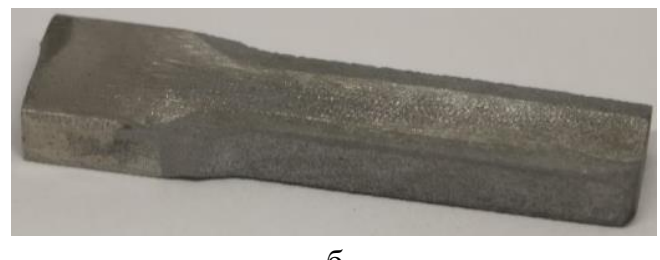

Рис. 5. Образец для исследования собственных частот колебаний без покрытия (а) и с нанесенным уплотнительным покрытием KHA-82+CoNiCrAlY (б)

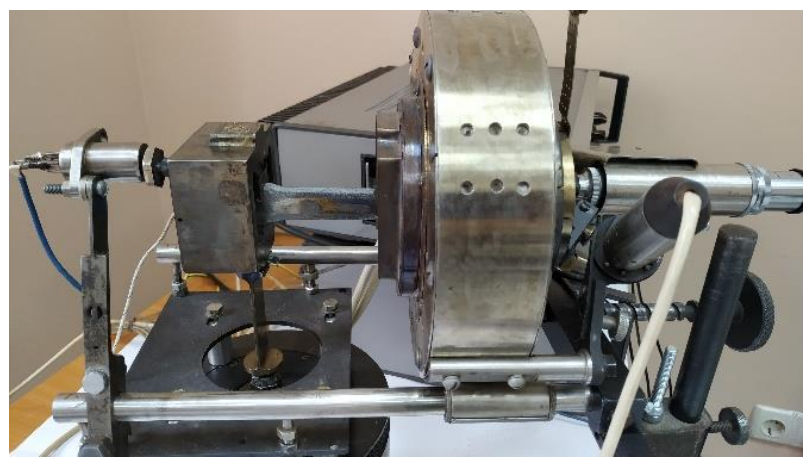

Рис. 6. Общий вид установки для определения собственных частот колебаний образцов

Нагружение образов выполняли путем задания гармонических колебаний защемленного конца посредством пьезоэлектрического преобразователя. Амплитуду колебаний контролировали при помощи оптического микроскопа и датчика обратной связи отслеживающего перемещения захвата образцов. В процессе определения собственной частоты амплитуду колебаний консольного конца образца под- 
бирали таким образом, чтобы обеспечить максимальные напряжения в образце на превышающие $50 \%$ от предела выносливости.

Это обеспечивало определение собственных частот колебаний с высокой точностью и не приводило к разрушению образцов. Температуру образца контролировали при помощи термопары, приваренной к зоне концентрации напряжений с точностью $\pm 2^{\circ} \mathrm{C}$. Образец выдерживали при заданной температуре в течение 5...7 мин, что обеспечивало его прогрев в рабочем сечении.

Частоту колебаний образца изменяли путём управления частотой возбуждения пьезоэлектрического вибратора. На первом этапе выполняли нагружение в широком частотном диапазоне, что позволило оценить собственную частоту образца по второй изгибной форме (рис. 7, а). На втором этапе выполняли трехкратное нагружение с записью амплитудно-частотной характеристики в узком диапазоне, охватывающем собственную частоту (рис. 7, б).

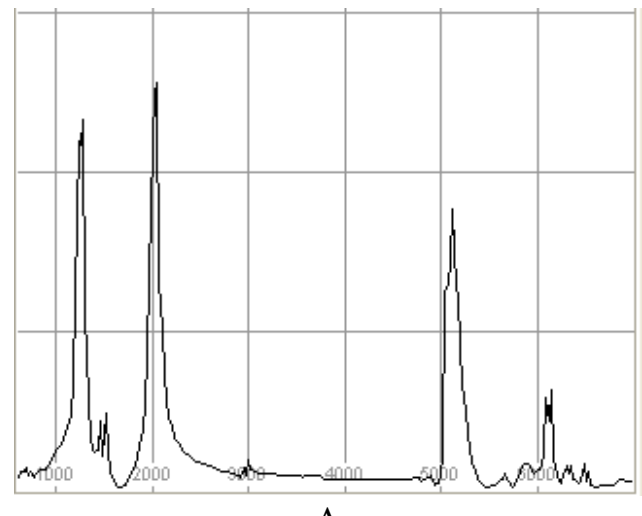

A

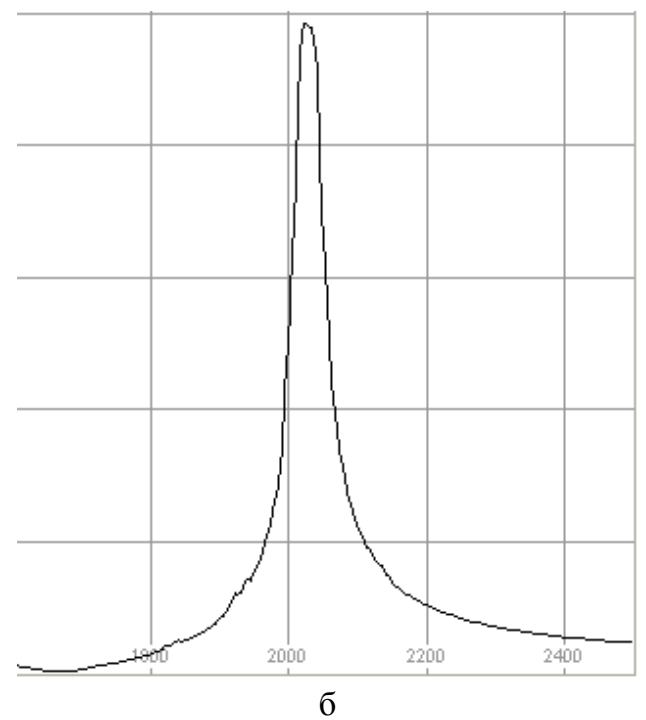

Рис. 7. Амплитудно-частотна диаграмма для исследуемого образца в диапазоне частот $600 \ldots 7000$ Гц (а) и $600 \ldots 2500$ Гц (б)

\section{Результаты исследования и их анализ}

Разрабатываемая методология определения динамического модуля упругости покрытия состоит из следующих основных этапов: определения собственной частоты колебаний образца с покрытием при различных температурах численным методом, установления зависимости динамического модуля упругости от температуры и частоты собственных колебаний, экспериментального определения собственной частоты колебаний образцов с покрытием при различных температурах и расчета динамического модуля упругости покрытия.

Моделирование колебания образцов выполняли для диапазона изменения модуля упругости покрытия от $1 \cdot 10^{10}$ до $1 \cdot 10^{11}$ Па и температуры от 20 до $1000^{\circ} \mathrm{C}$ численным методом.

Учитывая, что на собственную частоту образца с покрытием оказывает влияние как изменение его модуля упругости от температуры, так и изменение жесткости посредством термического расширения, выполняли анализ влияния указанных факторов. Установлено (рис. 8), что 79\% изменения собственной частоты колебаний при изменении температуры объясняется модулем упругости покрытия в то время как $21 \%$ изменений объясняется изменением ТКЛР. При этом увеличение модуля упругости приводит к увеличению собственной частоты, в то время как влияние ТКЛР обратное.

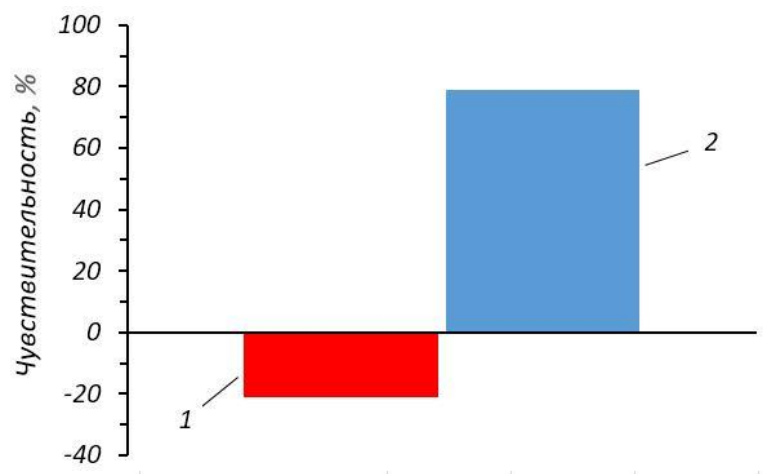

Рис. 8. Диаграмма влияния на изменение собственной частоты коэффициента термического расширения покрытий и модуля упругости; 1 - ТКЛР; 2 - модуль упругости

На основании данных, полученных в результате реализации плана численного эксперимента, построена поверхность (рис. 9), связывающая величину динамического модуля упругости уплотнительного покрытия в зависимости от температуры с резонансной частотой колебаний образца. Уравнение (1) описывающее указанную поверхность, позволяет решать обратную задачу и на основании экспериментальных данных измерения частоты собствен- 
ных колебаний образцов исследованной геометрии рассчитывать динамический модуль упругости покрытия.

$$
\begin{aligned}
& \mathrm{E}_{\text {покр }}^{\text {дин }}=-3,413 \cdot 10^{10}+9,068 \cdot 10^{6} \cdot \mathrm{T}+ \\
& +4,853 \cdot 10^{3} \cdot \mathrm{T}^{2}-1,562 \cdot 10^{7} \cdot \mathrm{f}_{\text {рез }}+ \\
& \quad+1,864 \cdot 10^{4} \cdot \mathrm{f}_{\text {рез }}^{2}, \text { Па }
\end{aligned}
$$

где Т -температура образца, ${ }^{\circ} \mathrm{C}$;

$\mathrm{f}_{\text {рез }}-$ резонансная частота, Гц.

Тесная корреляционная связь предсказанных с использованием уравнения (1) значений модуля упругости с экспериментальными значениями $(\mathrm{R}=0,99)$ указывает на удовлетворительное описание расчетных значений, полученных численным методом, полиномом второй степени. Таким образом можно утверждать, что разработанная методология позволяет надежно оценивать величину динамического модуля покрытий с использованием уравнения (1).

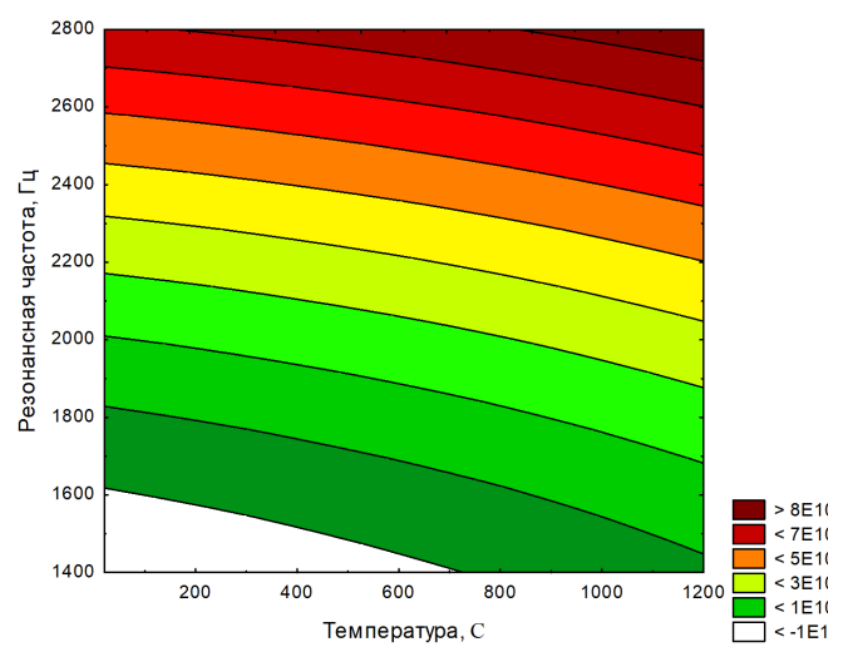

Рис. 9. Изолинии поверхности динамического модуля упругости уплотнительного покрытия в зависимости от температуры и резонансной частоты образца

Экспериментальная апробация разработанной методики на покрытии КНА-82+CoNiCrAlY подвергнутоv предварительной термической обработке и циклическому нагружению показала, что порядок величины его динамического модуля упругости хорошо согласуется со значением модуля упругости приведенном в работах $[5,10,13]$.

Различия в величинах могут прежде всего объясняться различиями в составе покрытия, его структуре, методе определения модуля упругости. Для исследуемого покрытия установлено наличие температурного гистерезиса модуля динамической упругости (рис. 10), что, вероятно, может быть связано с явлением циклического упрочения (разупрочнения) материала.

\section{Общие выводы и перспективы дальнейших исследований}

Таким образом, на основании реализации плана численного эксперимента по определению частоты собственных колебаний образцов, с нанесенным прирабатываемым покрытием при варьировании модуля упругости покрытия и температуры, а также решения обратной задачи, связанной с установлением зависимости модуля динамической упругости от частоты собственных колебаний образца с покрытием, позволили разработать расчетно-экспериментальную методику определения динамического модуля упругости прирабатываемых уплотнительных покрытий турбин ГТД. Ее реализация выполняется путем нанесения на верхнюю и нижние поверхности рабочей части образца заданной геометрии покрытия и экспериментального определения частоты его собственных колебаний в исследуемом диапазоне температур. Дальнейшее использование установленной зависимости модуля упругости от температуры и частоты собственных колебаний позволяет надежно и достоверно определить динамический модуль упругости покрытия.

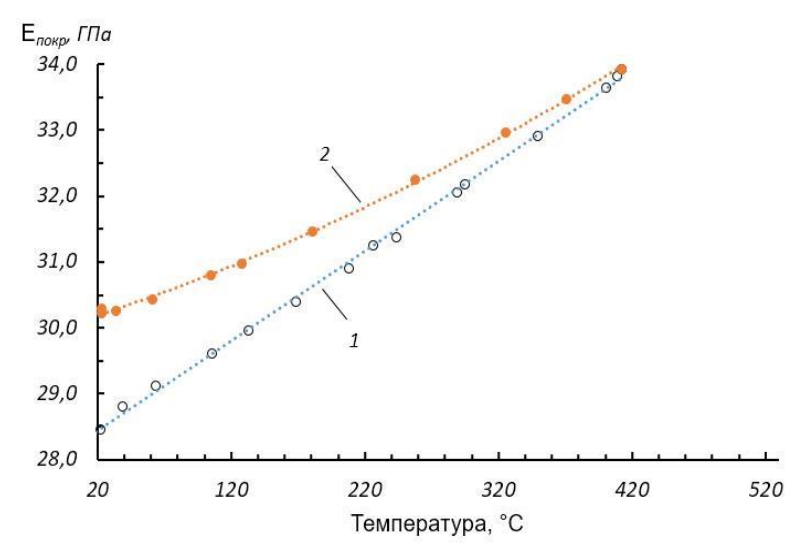

Рис. 10. Зависимость динамического модуля упругости уплотнительного покрытия

KHA-82+CoNiCrAlY после термической обработки, от температуры: 1 - нагрев; 2 - охлаждение

Перспективой дальнейших исследований с применением разработанной расчетно-экспериментальной методики могут являться исследования прирабатываемых уплотнительных покрытий, имеющих различный химический состав, структуру и свойства. Проведение таких исследований позволит оценить природу циклического изменения их свойств, а также выполнить оптимизацию состава и 
структуры покрытий. В свою очередь оптимизация покрытий по критерию соответствия модуля упругости покрытия основе позволит снизить величину напряжений на границе раздела покрытие-основа и повысит их адгезионную прочность.

\section{Литература}

1. Федотов, А. Ф. Прогнозирование эффективных модулей упругости пористых композиционных материалов [Текст] / А. Ф. Федотов // Известия вузов. Порошковая металлургия и функииональные покрытия. - 2015. - № 1. - С. 32-37.

2. Поляков, В. В. Модули упругости пористых псевдосплавов [Текст] / В. В. Поляков, А. В. Егоров, B. А. Турецкий // Известия Алтайского государственного университета. - 2004. - № 1. - С. 119 121.

3. Методы расчета механических характеристик пороматериалов малой плотности (обзор) [Текст] / Д. А. Черноус, Е. М. Петроковец, Д. А. Конек, С. В. Шилько // Механика композиционных материалов и конструкичй. - 2001. - T. 7, № 4. - С. 533-545.

4. Игнатьков, Д. А. Определение характеристик упругости неоднородных материалов динамическим методом[Текст] / Д. А. Игнатьков // Электронная обработка материалов. - 2011. - № 47(1). - C. 53-62.

5. Зеленина, E. А. Метод расчета физикомеханических характеристик плазменного покрытия на подложке при испытании образиов на изгиб [Текст] / Е. А. Зеленина, С. В. Лоскутов, А. В. Ершов // Нові матеріали і технології в металургї та машинобудуванні. - 2016. - № 2. - C. 107-110.

6. Determination Of Thermal Sprayed Coatings Elastic Modulus Using Four Point Bending Test [Text] / M. Beghinf, L. Bertini, F. Frendo, E. Giorni // WIT Transactions on Engineering Sciences. - 1997. - Vol. 17. - P. 61-70.

7. Low elastic modulus metals for joint prosthesis: Tantalum and nickel-titanium foams [Text] / M. Arciniegas, C. Aparicio, J. Manero, F. J. Gil // Journal of the European Ceramic Society. - 2007. - № 27(11). - P. 3391-3398.

DOI: 10.1016/j.jeurceramsoc.2007.02.184.

8. Nanoindentation investigation of the Young's modulus of porous silicon[Text] / D. Bellet, $P$.Lamagnère, A. Vincent, Y. Bréchet // Journal of Applied Physics. - 1996. - № 80(7). - P. 3772-3776.

DOI: 10.1063/1.363305.

9. Duan, K. Influence of sample deformation and porosity on mechanical properties by instrumented microindentation technique [Text] / K. Duan, R.W. Steinbrech // Journal of the European Ceramic Society.
- 1998. - No. 18(2). - P. 87-93. DOI: 10.1016/s09552219(97)00088-5.

10. Серенко, А. Н. Модуль упругости металла покрытия [Текст] / А. Н. Серенко, В. А. Роянов, C. В. Захаров // Вестник Приазовского гостехуниверситета : Сб. науч. трудов. - Мариуполь, 1999. - № 8. - C. 130-133.

11. Azarmi, F. Young's modulus measurement and study of the relationship between mechanical properties and microstructure of air plasma sprayed alloy 625 [Text] / F. Azarmi, T. Coyle, J. Mostaghim // Surface and Coatings Technolog. - 2009. - № 203(8). - P. 1045-1054. DOI: 10.1016/j.surfcoat.2008.09.035.

12. Tensile properties of a non-lineof-sight processed $\beta-\gamma-\gamma$ ' MCrAlY coating at high temperature [Text] / D. Texier, D. Monceau, F. Crabos, E. Andrieu // Surface and Coatings Technology. - 2017. - № 326, Part A. - P. 28-36.

DOI: 10.1016/j.surfcoat.2017.07.026.

13. Mechanical properties of porous titanium compacts prepared by powder sintering [Text] / I.-H. Oh, N. Nomura, N. Masahashi, S. Hanada // Scripta Materialia. - 2003. - № 49(12). - P. 1197-1202. DOI: 10.1016/j.scriptamat.2003.08.018.

\section{References}

1. Fedotov, A. F. Prognozirovanie jeffektivnyh modulej uprugosti poristyh kompozicionnyh materialov [Prediction of effective moduli of elasticity of porous composite materials]. Izvestija vuzov. Poroshkovaja metallurgija i funkcional'nye pokrytija, 2015, no. 1. pp. 32-37.

2. Poljakov, V. V., Egorov, A. V., Tureckij, V. A. Moduli uprugosti poristyh psevdosplavov [Elastic moduls of porous pseudoalloy]. Izvestija Altajskogo gosudarstvennogo universiteta, 2004, no. 1, pp. 119-121.

3. Chernous, D. A., Petrokovec, E. M., Konek, D. A., Shil'ko, S. V. Metody rascheta mehanicheskih harakteristik poromaterialov maloj plotnosti (obzor) [Methods for calculating the mechanical characteristics of low density porous materials (review)]. Mehanika kompozicionnyh materialov i konstrukcij, 2001, vol. 7, no. 4, pp. 533-545.

4. Ignat'kov, D. A. Opredelenie harakteristik uprugosti neodnorodnyh materialov dinamicheskim metodom [Determination of elastic characteristics of heterogeneous materials by the dynamic method]. Elektronnaja obrabotka materialov, 2011, no. 47(1), pp. 53-62.

5. Zelenina, E. A., Loskutov, S. V., Ershov, A. V. Metod rascheta fiziko-mehanicheskih harakteristik plazmennogo pokrytija na podlozhke pri ispytanii obrazcov na izgib [Method for calculating the physicomechanical characteristics of a plasma coating on a 
substrate when testing bending specimens]. Novi materiali $i$ tehnologii $v$ metalurgii ta mashinobuduvanni, 2016, no. 2, pp. 107-110.

6. Beghinf, M., Bertini, L., Frendo, F., Giorni, E. Determination Of Thermal Sprayed Coatings Elastic Modulus Using Four Point Bending Test. WIT Transactions on Engineering Sciences, 1997, vol. 17, pp. 61-70.

7. Arciniegas, M., Aparicio, C., Manero, J., Gil, F. J. Low elastic modulus metals for joint prosthesis: Tantalum and nickel-titanium foams. Journal of the European Ceramic Society, 2007, no. 27(11), pp. 33913398. DOI: 10.1016/j.jeurceramsoc.2007.02.184.

8. Bellet, D., Lamagnère, P., Vincent, A., Bréchet, Y. Nanoindentation investigation of the Young's modulus of porous silicon. Journal of Applied Physics, 1996, no. 80(7), pp. 3772-3776. DOI: 10.1063/1.363305.

9. Duan, K., Steinbrech, R. W. Influence of sample deformation and porosity on mechanical properties by instrumented microindentation technique. Journal of the European Ceramic Society, 1998, no. 18(2), pp. 8793. DOI: 10.1016/s0955-2219(97)00088-5.
10. Serenko, A. N., Rojanov, V. A., Zaharov, S. V. Modul' uprugosti metalla pokrytija [The modulus of elasticity of the metal coating]. Vestnik Priazovskogo gostehuniversiteta : Sb. nauch. trudov. Mariupol', 1999, no. 8, pp. 130-133.

11. Azarmi, F., Coyle, T., Mostaghim, J. Young’s modulus measurement and study of the relationship between mechanical properties and microstructure of air plasma sprayed alloy 625. Surface and Coatings Technolog, 2009, no. 203(8), pp. 1045-1054. DOI: 10.1016/j.surfcoat.2008.09.035.

12. Texier, D., Monceau, D., Crabos, F., Andrieu, E. Tensile properties of a non-lineof-sight processed $\beta$ $\gamma-\gamma$ ' MCrAlY coating at high temperature. Surface and Coatings Technology, 2017, no. 326, Part A, pp. 28-36. DOI: 10.1016/j.surfcoat.2017.07.026.

13. Oh, I.-H., Nomura, N., Masahashi, N., Hanada, $\mathrm{S}$. Mechanical properties of porous titanium compacts prepared by powder sintering. Scripta Materialia, 2003, no. 49(12), pp. 1197-1202.

DOI: $10.1016 /$ j.scriptamat.2003.08.018.

Поступила в редакцию 5.06.2019, рассмотрена на редколлегии 7.08.2019

\section{РОЗРАХУНКОВО-ЕКСПЕРИМЕНТАЛЬНА МЕТОДИКА ВИЗНАЧЕННЯ ДИНАМІЧНОГО МОДУЛЯ ПРУЖНОСТІ ПРИРОБЛЮВАНИХ УЩІЛЬНЮВАЛЬНИХ ПОКРИТТІВ ТУРБІН ГТД}

\section{В. Л. Грешта, Д. В. Павленко, Я. В. Двірник, Д. В. Ткач}

Метою роботи було розробка та апробація методики визначення динамічного модуля пружності прироблюваних ущільнювальних покриттів турбін газотурбінних двигунів (ГТД). До даних покриттів висувається ряд неоднозначних вимог, тому для їх задоволення запропоновано застосовувати покриття зі змінними властивостями на різних етапах життєвого циклу газотурбінних двигунів. Однак розробка нових покриттів вимагає проведення різного роду механічних випробувань, в тому числі і для оцінки динамічного модуля пружності. Пориста структура і, відповідно, низька міцність розроблюваних покриттів не дозволяє застосовувати стандартні методи оцінки механічних властивостей, тому виникла необхідність розробки спеціальної методики визначення модуля пружності. У процесі дослідження були застосовані метод кінцевих елементів, статистичні методи, експериментальні методи визначення власної частоти коливань. Дослідження виконували для прироблюваних ущільнювальних покриттів статору турбін газотурбінних двигунів КНА-82 + CoNiCrAlY. Чисельний експеримент було виконано в програмному комплексі Ansys Workbench 2019 R2. Оскільки покриття застосовуються в умовах підвищених температур необхідно було оцінювати модуль пружності при різних температурах, що вимагало проведення додаткових досліджень температурозалежних властивостей, що впливають на досліджувану величину. В результаті реалізації плану чисельного експерименту по визначенню частоти власних коливань зразків, з нанесеним покриттям при варіюванні його модуля пружності і температури, а також розв'язання оберненої задачі встановлення залежності модуля динамічної пружності від частоти власних коливань зразка з покриттям, розробили розрахунково-експериментальну методику визначення динамічного модуля пружності прироблюваних ущільнювальних покриттів турбін ГТД. Розроблена методика застосовується для визначення динамічного модуля пружності прироблюваних покриттів різного хімічного складу і структури в області робочих температур, що може бути використана для оптимізації їх складу, структури і властивостей.

Ключові слова: план експерименту; модуль пружності; прироблювані покриття; газотурбінний двигун; методика; власна частота; метод кінцевих елементів; модуль пружності. 


\section{DESIGN-EXPERIMENTAL TECHNIQUE FOR DETERMINING A DYNAMIC MODEL OF ELASTICITY OF PRODUCED SEALING COATINGS OF GTE TURBINES}

\section{L. Greshta, D. V. Pavlenko, Ya. V. Dvirnyk, D. V. Tkach}

The aim of the work was the development and testing of a method for determining the dynamic modulus of elasticity of running-in sealing gasket coatings for GTE turbines. Many contradictory requirements are put forward to these coatings, therefore, to satisfy them, it was proposed to apply coatings with variable properties at various stages of the life cycle of gas turbine engines. However, the development of new coatings requires a variety of mechanical tests, including to evaluate the dynamic modulus of elasticity. The porous structure and, accordingly, the low strength of the developed coatings do not allow the use of standard methods for the evaluation of mechanical properties, so there is a need to develop a special method for determining the elastic modulus. In the course of the study, the finite element method, statistical methods, experimental methods for determining the natural frequency of oscillations were applied. Investigations were carried out for running-in sealing coating of the stator of turbines of gas turbine engines KNA-82 + CoNiCrAlY. The numerical experiment was performed in the Ansys Work-bench 2019 R2 software package. Since coatings are used at elevated temperatures, it was necessary to estimate the modulus of elasticity at various temperatures, which required additional studies of temperature-dependent properties that affect the desired value. As a result of the implementation of the plan of a numerical experiment to determine the frequency of natural oscillations of samples with a coating while varying its elastic modulus and temperature, as well as solving the inverse problem of establishing the dependence of the dynamic elastic modulus on the natural oscillation frequency of a coated sample, we developed a calculation and experimental method for determining the dynamic modulus elasticity of running-in sealing coatings of GTE turbines. The developed technique is used to determine the dynamic modulus of elasticity of running-in coatings of different chemical composition and structure in the range of operating temperatures, which can be used to optimize their composition, structure, and properties.

Keywords: experimental design; modulus of elasticity; surface-to-be-coated coating; gas turbine engine; method; natural frequency; finite element method; modulus of elasticity.

Грешта Виктор Леонидович - канд. техн. наук, профессор кафедры физического материаловедения Запорожского национального технического университета, Украина.

Павленко Дмитрий Викторович - канд. техн. наук, доцент, профессор кафедры технологии авиационных двигателей Национального университета "Запорожская политехника"; ведущий инженер ГП "Ивченко-Прогресс", Украина.

Двирнык Ярослав Викторович - канд. техн. наук, начальник отдела АО "МОТОР СИЧ", Украина.

Ткач Дарья Владимировна - канд. техн. наук, доцент кафедры физического материаловедения Запорожского национального технического университета, Украина.

Greshta Viktor Leonidovich - Candidate of Technical Science, Professor of Dept. of Physical materials science, Zaporizhzhya National Technical University, Ukraine,

e-mail: kafedra_fm@zntu.edu.ua, ORCID Author ID: 0000-0002-4589-6811.

Pavlenko Dmytro Viktorovich - Candidate of Technical Science, associate professor, professor of department of Aircraft engines technologies, National University "Zaporizhzhia polytechnic"; leading engineer of SE "Ivchenko-Progress", e-mail: dvp1977dvp@gmail.com, ORCID Author ID: 0000-0001-6376-2879.

Dvirnyk Yaroslav Viktorovich - Candidate of Technical Science, Head of Department of Motor Sich JSC, e-mail: dvirnyk@gmail.com, ORCID Author ID: 0000-0001-5439-5413.

Tkach Daria Vladimirovna - Candidate of Technical Science, Assistant Professor of Dept. of Physical materials science, Zaporizhzhya National Technical University, Ukraine,

e-mail: odarka_ua@hotmail.com, ORCID Author ID: 0000-0003-0851-1481. 